\title{
Testes-specific hemoglobins in Drosophila evolved by a combination of sub- and neofunctionalization after gene duplication
}

\author{
Eva Gleixner ${ }^{1,2}$, Holger Herlyn ${ }^{3}$, Stefan Zimmerling ${ }^{1}$, Thorsten Burmester ${ }^{4}$ and Thomas Hankeln ${ }^{1 *}$
}

\begin{abstract}
Background: For a long time the presence of respiratory proteins in most insects has been considered unnecessary. However, in recent years it has become evident that globins belong to the standard repertoire of the insect genome. Like most other insect globins, the glob1 gene of Drosophila melanogaster displays a conserved expression pattern in the tracheae, the fat body and the Malpighian tubules.
\end{abstract}

Results: Here we show that the recently discovered D. melanogaster globin genes glob2 and glob3 both display an unusual male-specific expression in the reproductive tract during spermatogenesis. Both paralogs are transcribed at equivalent mRNA levels and largely overlap in their cellular expression patterns during spermatogenesis.

Phylogenetic analyses showed that glob2 and glob3 reflect a gene duplication event that occurred in the ancestor of the Sophophora subgenus at least 40 million years ago. Therefore, flies of the Drosophila subgenus harbor only one glob2/3-like gene.

Conclusions: Phylogenetic and sequence analyses indicate an evolution of the glob2 and glob3 duplicates by a combination of sub- and neofunctionalization. Considering their restricted, testes-specific expression, an involvement of both globins in alleviating oxidative stress during spermatogenesis is conceivable.

\section{Background}

$\mathrm{O}_{2}$ supply in insects is mainly accomplished by the highly specialized and effective tracheal system. Respiratory proteins like hemoglobins (Hbs) or hemocyanins had long been considered dispensable in insects [1,2]. Only a few taxa that are specifically adapted to a hypoxic environment were considered as exceptions [3]. Among these, the larvae of the horse botfly Gasterophilus intestinalis and backswimmers of the genus Anisops possess intracellular $\mathrm{Hbs}$, which probably carry out myoglobin-like $\mathrm{O}_{2}$-storage functions [3-6]. Extracellular Hbs for oxygen transport and storage are present in the hemolymph of the hypoxia-tolerant aquatic larvae of chironomid midges $[7,8]$.

In recent years, genomic sequence data have provided evidence that $\mathrm{Hb}$ genes are indeed a standard component of the insect genome [9]. Recent discoveries of Hbs in insects include the honeybee Apis mellifera [10], the

\footnotetext{
* Correspondence: hankeln@uni-mainz.de

${ }^{1}$ Institute of Molecular Genetics, University of Mainz, 55099 Mainz, Germany Full list of author information is available at the end of the article
}

mosquitoes Anopheles gambiae and Aedes aegypti [11], and other dipteran, lepidopteran, coleopteran and hymenopteran species $[9,12]$. The insect model organism Drosophila melanogaster was initially shown to possess an $\mathrm{Hb}$ gene named glob1 (CG9734) [13]. Glob1 is predominantly expressed at substantial amounts in the fat body and the tracheal system of Drosophila embryos, larvae and adults [14]. These expression sites, which appear to be conserved features of Hbs in other insect species as well, suggest that glob1 function is associated with oxidative metabolism $[9,14]$. The intracellular glob1 protein binds $\mathrm{O}_{2}$ at high affinities $\left(\mathrm{P}_{50}\left(\mathrm{O}_{2}\right)=0.12-0.15\right.$ Torr $)$ and forms a typical globin fold, in which the heme iron atom is hexacoordinated $[14,15]$. The glob1 gene is downregulated upon experimental hypoxia in vitro and in vivo [16,17], while hyperoxia and intermittent hypoxic regimes trigger a slight transcriptional upregulation. These data indirectly suggest that glob1 might also be instrumental in binding excess $\mathrm{O}_{2}$ or noxious reactive oxygen species (ROS) in the tracheal system [17].
C Biomed Central

(c) 2012 Gleixner et al; licensee BioMed Central Ltd. This is an Open Access article distributed under the terms of the Creative Commons Attribution License (http://creativecommons.org/licenses/by/2.0), which permits unrestricted use, distribution, and reproduction in any medium, provided the original work is properly cited. 
More recently, two additional globin genes referred to as glob2 (CG15180) and glob3 (CG14675) were identified in the Drosophila genome [18]. These two genes represent related paralogous copies, which in D. melanogaster both reside on chromosome 3R about $800 \mathrm{~kb}$ apart in head-to-tail orientation. Phylogenetic analyses showed that glob2 and glob3 are only distantly related to glob1 and most other insect $\mathrm{Hb}$ genes [18]. The basal position of glob2 and 3 in the insect $\mathrm{Hb}$ phylogenetic tree and the monophyly of the two duplicates were further substantiated by their exon-intron pattern [18].

Conceptual translations of $D$. melanogaster glob2 and glob3 result in proteins of 222 and 195 amino acids, respectively, thus exceeding the typical globin length of about 140-150 amino acids due to $\mathrm{N}$ - and C-terminal extensions. In the globin fold, however, amino acid residues functionally important for heme and ligand binding (e.g. the PheCD1 and the proximal and distal histidines E7 and F8) are well conserved in the proteins. In our initial analysis [18], glob2 appeared to be expressed at a much lower level than glob1 as evidenced by only a few corresponding expression sequence tag (EST) entries in databases, while glob3 lacked EST transcriptional evidence. To study the functional roles of glob2 and 3 we now conducted a more detailed expression analysis of both genes across Drosophila developmental stages, gender and tissues. In addition, the availability of completely sequenced Drosophila genomes representing nine species of the Sophophora subgenus and three species of the Drosophila subgenus [19] allowed us to gain novel insight into the molecular evolution and phylogeny of the glob2 and glob3 paralogs, which is relevant for functional interpretations.

\section{Results}

Genomic organization of glob2 and glob3 paralogs in Drosophila

While the glob2 and glob3 gene pair is present in the nine Drosophila species belonging to the Sophophora subgenus, the three species belonging to the Drosophila subgenus harbour only one co-ortholog, which we name glob2/3 to reflect the uncertain orthology relationship (Figure 1; for gene designations, see Additional File 1). The genes located 5' of glob3 and glob2/3 vary across the investigated Drosophila species. In contrast, the 3' genomic environment of glob3 and glob2/3 is conserved throughout the taxon sample. In all twelve fly genomes studied glob3 and glob2/3 are located on the Muller element $\mathrm{E}$, which is equivalent to chromosome $3 \mathrm{R}$ in $D$. melanogaster. Chromosomal location and synteny relationships are more complex for glob2: D. ananassae and D. pseudoobscura/persimilis harbor glob2 and glob3 as tandem duplicates in head-to-tail orientation with about 500 bp space between the gene copies. This glob2-glob3

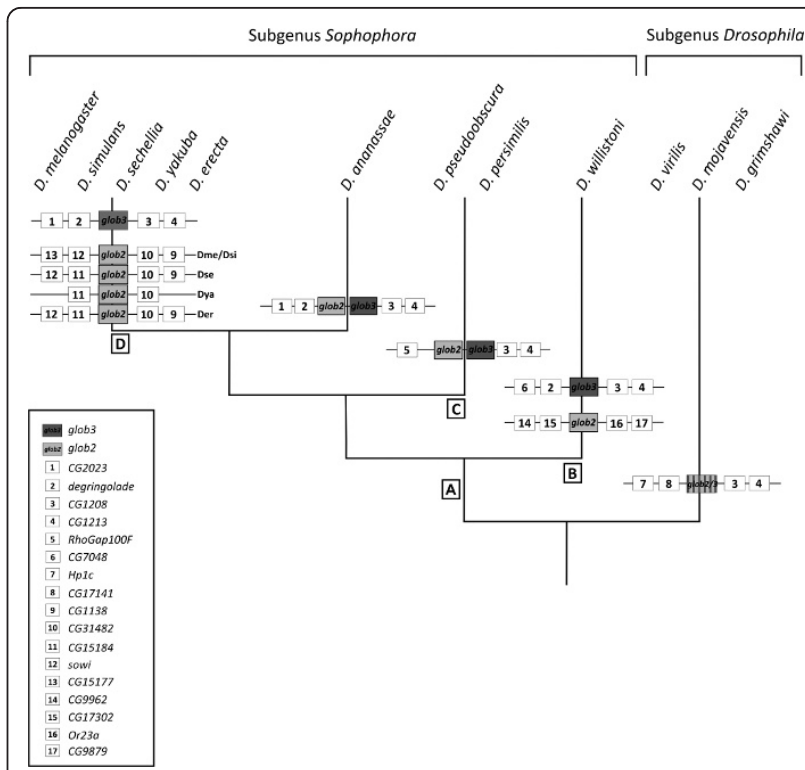

Figure 1 Hypothetical scenario of evolutionary events leading to glob2 and glob3 genes in drosophilids. The chromosomal localization and organisation of glob2, glob3 and glob2/3 genes and flanking regions in different subgroups of Drosophila phylogeny are displayed. [A] tandem duplication of glob2/3 gene; [B] separation of glob2 and glob 3 by transposition of glob 2 to Chr 2R (= Dme Chr $2 \mathrm{~L}) ;[\mathrm{C}]$ tandem glob2 and glob3, but chromosome breakage distal to the glob2-glob3 tandem; [D] transposition of glob2 to 3R 83 F4, $800 \mathrm{~kb}$ apart from glob3.

tandem resides on a chromosome arm, which is equivalent to arm 3R in D. melanogaster (Figure 1). In D. willistoni, which branches off even closer to the base of the Sophophora subgenus, the glob2 copy is located on arm $2 \mathrm{R}$ corresponding to $2 \mathrm{~L}$ in $D$. melanogaster (Figure 1 ). In the representatives of the $D$. melanogaster subgroup, glob2 and glob3 are linked on arm 3R, but separated by about $800 \mathrm{~kb}$.

\section{Reconstruction of a globin phylogenetic tree}

Pairwise sequence comparisons of nucleotide sequences using Geneconv did not detect segments of high similarity in the complete dataset comprising 35 globin sequences. Thus subsequent analyses should not be impaired by concerted sequence evolution of paralogs within species. ML based tree reconstruction revealed a monophyletic origin of glob2 and glob3 sequences of the genus Drosophila (LR-ELW 99) under exclusion of glob1 sequences (Additional File 2A). Within this clade, Sophophora glob2 and glob3 each constitute a monophylum with moderate support (LR-ELW 83) under exclusion of glob2/3 from the Drosophila subgenus. Among the representatives of the Sophophora subgenus, the observed phylogeny largely corresponds to the accepted species phylogeny introduced by Clark et al. [19]. Bayesian inference essentially confirmed the ML based 
phylogeny, although the support for a monophyletic Sophophora glob2-glob3 clade was low (Additional File 2B).

Branch lengths estimated by ML and Bayesian tree inference consistently illustrate that glob1 orthologs diverged with lower nucleotide substitution rates than the glob2 and glob 3 orthologs. This conclusion is supported by amino acid substitution rates inferred separately for the glob1, glob2 and glob3 clades from pairwise within-group sequence comparisons using a PAM substitution matrix and assuming the Drosophila divergence times suggested by Tamura et al. [20]. In doing so, we calculated an average of $0.95 * 10^{-9}$ replacements per site per year for the glob1 clade and elevated rates of $2.8^{*} 10^{-9}$ and $3.5^{*} 10^{-9}$ replacements per site per year for the glob2 clade and glob3 clade, respectively. Within the glob2 and 3 clades, branch length estimates and results from molecular evolutionary sequence analysis (see below, LRTII) further suggest that Sophophora glob 2 is more derived than Sophophora glob 3, compared to the hypothetical ancestor of both gene lineages.

Parametric bootstrap testing as implemented in Treefinder identified a 'glob2/3 duplication-in-Sophophora subgenus' topology as the significantly better fit ( $\mathrm{p}=$ 0.96) of the data, compared to an alternative tree topology reflecting a 'glob2 or 3 deletion-in-Drosophila subgenus' scenario.

\section{Molecular evolution}

Due to consistent results from unconstraint tree reconstruction and hypotheses testing, we took the topology reflecting the 'glob2/3 duplication-in-Sophophora subgenus' scenario as template for subsequent analyses of globin sequence evolution. We thereby focused on the evolutionary fate of Sophophora glob2 and Sophophora glob3 after the presumed duplication of an ancestral glob2/3 gene in the Sophophora stem lineage. In order to minimize any potential confounding effect of mutational saturation on the results, we addressed this issue based on a restricted sample comprising three Drosophila glob2/3, nine Sophophora glob2, and nine Sophophora glob3 sequences (altogether 21 sequences).

The fit of alternative beta model M8 on the sequence dataset was identical, irrespective of the initial $d_{\mathrm{N}} / d_{\mathrm{S}}$ value chosen $(l=-4,398.493)$. As the fit of the beta null model M7 was in the same range $(l=-4,398.492)$, LRTI did not support the presence of positively selected sites across the sequence dataset $(2 \Delta l=0.002 ; \mathrm{df}=2$; $\left.\mathrm{cv}_{5 \%}=5.992 ; \mathrm{p}>>0.05\right)$. The prevalence of negative purifying selection was reflected by an M7 estimate for $d_{\mathrm{N}} / d_{\mathrm{S}}$ of 0.141 . Likewise, the alternative version of modified model A $(l=-4,451.778)$ and the null implementation of modified model A $(l=-4,451.808)$ explained the evolution of the dataset equally well, when specifying the Sophophora glob3 stem lineage as foreground. Consequently, LRTII did not support the presence of a positively selected extra site class across the Sophophora glob3 stem lineage $\left(2 \Delta l=0.060 ; \mathrm{df}=1 ; \mathrm{cv}_{5 \%}=2.71 ; \mathrm{p}\right.$ $>>0.05)$. However, the alternative version of modified model A was the significantly better fit $(l=-4,444.314)$, compared to the null version $(l=-4,446.723)$, when specifying the Sophophora glob2 stem lineage as foreground (LRTII: $2 \Delta l=4.818$; $\mathrm{df}=1 ; \mathrm{cv}_{2.5 \%}=3.84 ; \mathrm{p}<$ $0.025)$. This result thus even holds when lowering the $5 \%$ level of significance to $2.5 \%$ in order to correct for twofold testing. According to the alternative version of modified model A, a remarkably high proportion of ca. $42 \%$ of codon sites underwent positive selection $\left(d_{\mathrm{N}} / d_{\mathrm{S}}\right.$ $=5.642$ ) along the Sophophora glob2 stem lineage, but experienced negative selection or evolved neutrally across the background represented by all other branches of the phylogeny shown in Figure 2.

\section{Quantification of glob2, glob3 and glob2/3 mRNA expression}

In our earlier study, six EST entries in the database of D. melanogaster revealed that glob2 is actually expressed, albeit at a rather low level [18]. Seven additional D. melanogaster ESTs can now be reported (UniGene:EC252960, UniGene:EL878213, UniGene: EL878330, UniGene:EL878331, UniGene:EL878455, UniGene:EC067562, UniGene:EC061683), most of which are derived from adult flies. ESTs corresponding to glob2 are also present for $D$. simulans, $D$. sechellia and $D$. yakuba adults, and eight $D$. simulans ESTs derive from $3^{\text {rd }}$ instar larvae.

Because of the absence of glob3 EST entries for D. melanogaster and due to our initial failure to recover

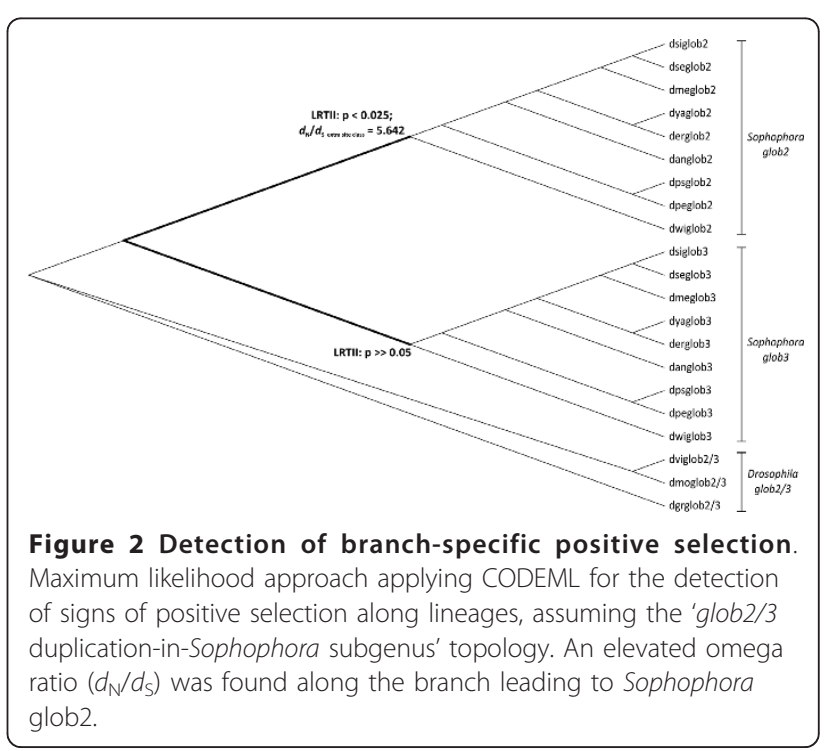


cDNA from larval or adult RNA by RT-PCR we (erroneously) assumed that glob3 is not expressed [18]. Since 2007, however, there are three glob3 ESTs reported for adult D. melanogaster (UniGene:EL876979, UniGene: EL877168, UniGene:EL877169). In addition, we could identify three ESTs from $D$. simulans (two from an adult, one from $3^{\text {rd }}$ instar larvae), three ESTs from adult D. sechellia and one EST from adult D. yakuba. Based on these findings, we systematically re-analyzed glob2, glob3 and glob2/3 mRNA expression by quantitative real-time RT-PCR in embryos, male and female larvae, pupae and adult males/females of $D$. melanogaster and the distantly related $D$. virilis.

In embryos, the mRNA expression of $D$. melanogaster glob2 was minimal (not shown). Dmeglob2 turned out to be maximally expressed in male adult flies (Figure 3A). Expression levels in male larvae and pupae were at $43 \%$ and $78 \%$ relative to male adults. In female larvae, pupae and adults dmeglob2 expression was always beyond the detection limit. The developmental expression pattern of dmeglob3 showed a high similarity to dmeglob2. Dmeglob3 mRNA expression in embryos (not shown) as well as in female larvae, pupae and adult flies was not detectable. The amounts of dmeglob3 mRNA in male larvae and pupae were about $16 \%$ and $50 \%$ of the maximal mRNA expression level obtained in male adult flies (Figure 3B). Thus, for both, dmeglob2 and glob3, we could experimentally confirm the expression pattern derived from the modENCODE project implemented in FlyBase http://flybase.org/reports/FBgn0037385.html; http://flybase.org/reports/FBgn0250846.html. The developmental expression pattern of the single-copy glob2/3 gene in $D$. virilis was generally matching those of the $D$. melanogaster paralogs. In embryos, no expression of dviglob2/3 expression could be measured (not shown), while adult males showed maximum expression (Figure 3C). Male and female larvae expressed $5 \%$, male pupae $38 \%$, and female pupae and adults $2 \%$ and $6 \%$ of the male adult level (Figure 3C).

We also compared absolute mRNA copy numbers and observed that D. melanogaster glob2 and glob3 expression in male adults was about the same level, with 144784 copies and 150026 copies of mRNA (per $50 \mathrm{ng}$ total RNA), respectively. The amount of D. virilis glob2/ 3 mRNA (311673 mRNA copies per 50 ng total RNA) in male adult flies was approximately equivalent to the sum of the D. melanogaster glob2 and glob3 mRNA copy numbers (Additional File 3).

With the analyses of the genomic region of D. melanogaster glob3, we identified a non-LTR-retrotransposable element (TE) of the Jockey family 234 bp upstream of the coding sequence of dmeglob3. The TE has a length of about $3.1 \mathrm{~kb}$ and is truncated at the 5 'end. It is located on the minus-strand, its 3'end exhibits an A-
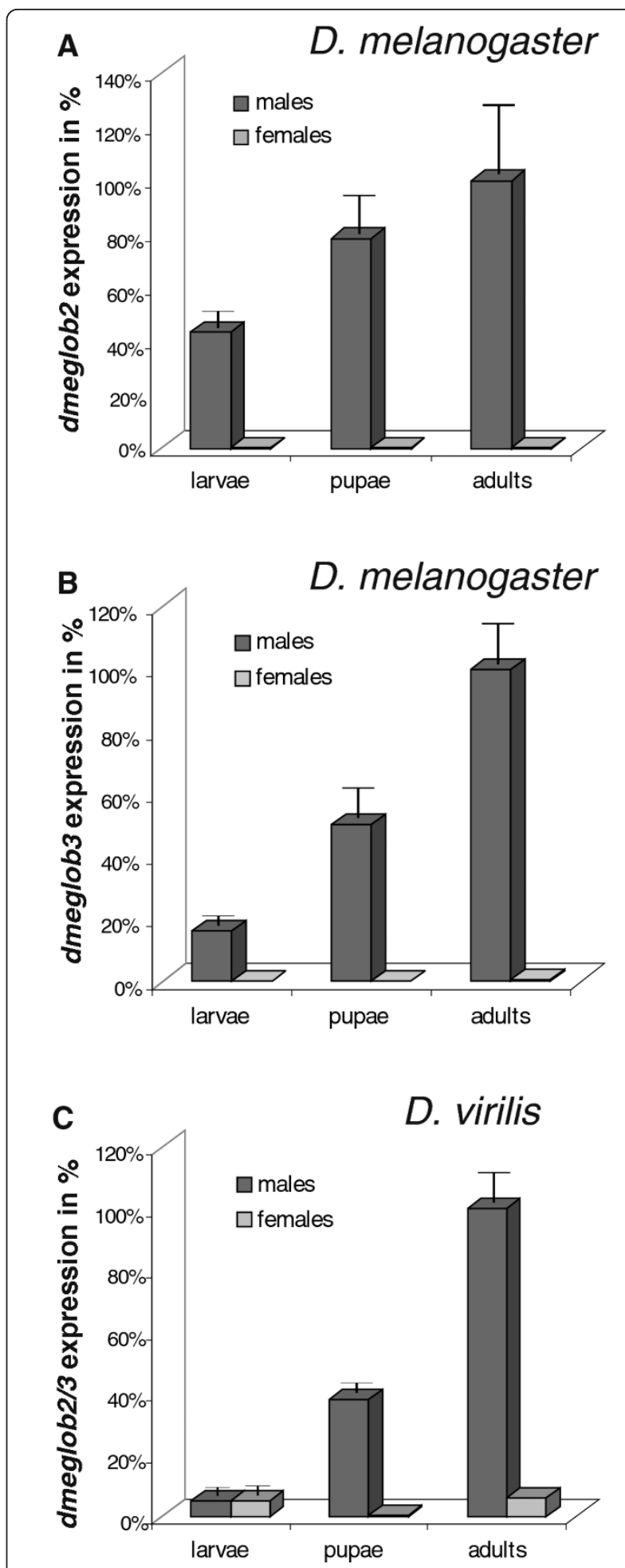

Figure 3 Dmeglob2, dmeglob3 and dviglob2/3 mRNA expression pattern. Quantification of mRNA expression of glob2 and glob3 in D. melanogaster and glob2/3 in D. virilis in sexed $3^{\text {rd }}$ instar larvae, pupae and adults. mRNA levels (bars) are shown relative to glob-expression in adult males. (A) glob2 expression in D. melanogaster. (B) glob3 expression in D. melanogaster. (C) glob2/3 expression in D. virilis. 
rich stretch and it is specific for D. melanogaster. By applying genomic PCR on different wild type strains of D. melanogaster (Oregon R, Canton S, Berlin and Acharron), we confirmed the fixation of the transposon in these wild-type strains. By comparison with genomic data from $D$. sechellia, we found that the retrotransposon ends $142 \mathrm{bp}$ upstream of the 5'UTR of dmeglob3 (Additional File 4). To find out if the retrotransposon interferes with the promoter of dmeglob3, we predicted possible transcription start sites applying the hidden Markov model-based program McPromoter [21,22] and the time-delayed neuron network based program NNPP $[23,24]$. Only the latter identified a candidate Pol II promoter for D. simulans glob3 and D. sechellia glob3 (but not for D. melanogaster glob2 and glob3), which is located $216 \mathrm{bp}$ upstream of the translation start-site and 124 bp upstream of the 5'UTR (of dmeglob3) spanning the corresponding integration site of the TE in D. melanogaster (Additional File 4).

\section{Tissue expression patterns of $g l o b 2$, glob3 and $g l o b 2 / 3$}

The expression patterns of glob2 and glob3 were examined in head, thorax and abdomen of D. melanogaster adult flies by mRNA in situ hybridization. In head and thorax, no staining could be observed for both globin genes (data not shown). In the abdomen, the only tissues stained were the testes from male flies, as already expected from the male expression levels in qPCR. The hybridization experiments were repeated on dissected testes of $D$. melanogaster and $D$. virilis. Staining signals indicated a glob2 expression in various regions of the $D$. melanogaster adult testes, corresponding to several stages of spermatogenesis. Hybridization signals were observed for primary spermatocytes, meiotic spermatocytes, round spermatids and early differentiating spermatids (Figure 4A). No staining was obtained in stem cells, mitotic cells, late elongating spermatids and

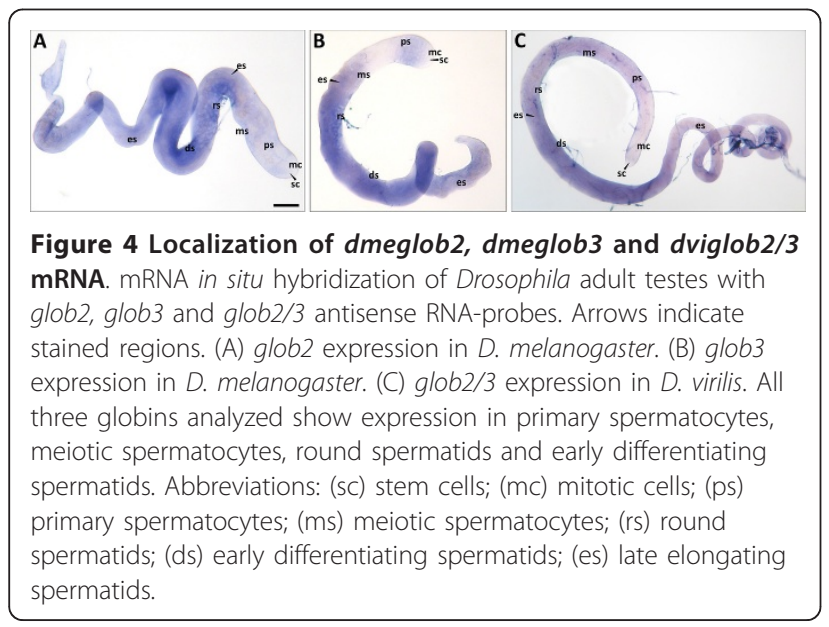

mature sperm. Glob3 in D. melanogaster exhibited a very similar expression pattern, although with a slightly weaker staining intensity (Figure 4B). In D. virilis, in situ hybridization with a glob2/3 antisense probe also resulted in very similar signals in primary spermatocytes, meiotic spermatocytes, round spermatids and early differentiating spermatids (Figure 4C). Sense probes, used as a negative control, did not show staining signal (Additional File 5).

\section{Regulation of glob2 and glob3 expression under hypoxia and hyperoxia}

D. melanogaster glob2 and glob3 mRNA levels were measured by qPCR in normoxic $\left(21 \% \mathrm{O}_{2}\right)$ and experimentally hypoxic as well as hyperoxic male adult $D$. melanogaster. $L D H$, analyzed as a positive control for hypoxia up-regulation via the HIF-1 pathway [25], showed the expected increase in mRNA expression (Additional File 6A+B).

A short-term severe hypoxia regime $\left(1 \% \mathrm{O}_{2}\right.$ for $1 \mathrm{~h}, 3$ $\mathrm{h}$ and $4 \mathrm{~h}$ ) resulted in a significant, gradual decrease of dmeglob2 mRNA levels to about $45 \%$ of the normoxic control (Figure 5A). Long-term moderate hypoxia (6\% $\mathrm{O}_{2}$ for $24 \mathrm{~h}$ ) triggered only a slight down-regulation of dmeglob2 mRNA expression (Figure 5B). Dmeglob3 mRNA expression was even more reduced (to $23 \%$ after $1 \% \mathrm{O}_{2}$ for $3 \mathrm{~h}$; Figure 5D). After long-term hypoxia (6\% $\mathrm{O}_{2}$ for $24 \mathrm{~h}$ ), dmeglob3 mRNA level decreased to about $60 \%$ compared to the normoxic control (Figure 5E). After hyperoxic treatment, no statistically significant
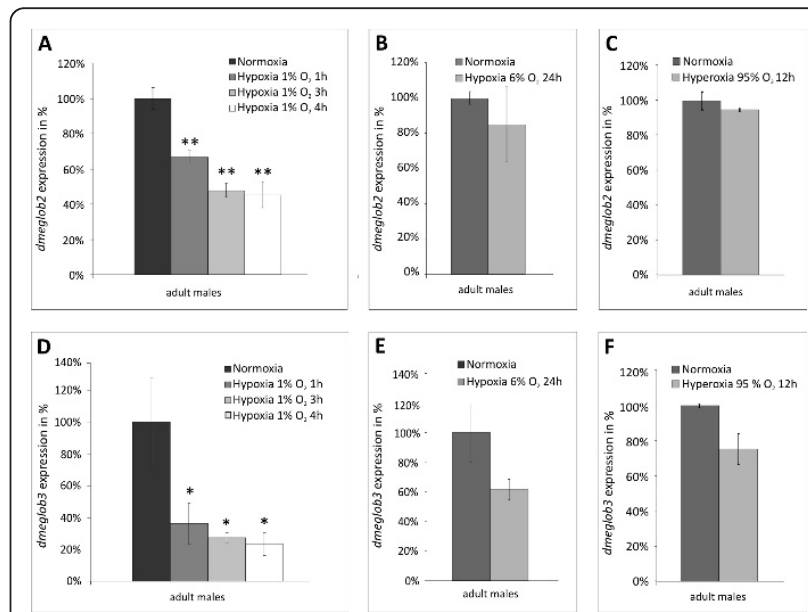

Figure 5 Regulation of dmeglob2 and dmeglob3 mRNA male adults after hypoxic and hyperoxic stress. mRNA levels (bars) are shown relative to the gene expression at normoxia (21\%). (A)

Dmeglob2 expression after $1 \% \mathrm{O}_{2}$ for $1 \mathrm{~h}, 3 \mathrm{~h}$ and $4 \mathrm{~h}$. (B) Dmeglob2 expression after $6 \% \mathrm{O}_{2}$ for $24 \mathrm{~h}$. (C) Dmeglob3 expression after $95 \%$

$\mathrm{O}_{2}$ for $12 \mathrm{~h}$. (D) Dmeglob3 expression after $1 \% \mathrm{O}_{2}$ for $1 \mathrm{~h}, 3 \mathrm{~h}$ and 4 h. (E) Dmeglob3 expression after $6 \% \mathrm{O}_{2}$ for $24 \mathrm{~h}$. (F) Dmeglob3 expression after $95 \% \mathrm{O}_{2}$ for $12 \mathrm{~h}\left({ }^{*} \mathrm{p}<0.05 ;{ }^{* *} \mathrm{p}<0.01\right)$. 
changes of mRNA levels of both, dmeglob2 and 3 were detected (Figure 5C+F). A bioinformatical search for hypoxiaresponsive elements (HRE) [26] in the twelve genomes, conducted by the rVISTA program [27]; http://rvista.dcode.org/), did not yield evidence for interspecifically conserved HRE motifs.

\section{Discussion}

\section{Globin expression in Drosophila testes: a novel or} traditional function?

Since the identification of a globin gene (glob1) in $D$. melanogaster [13] it has become clear that globins belong to the standard repertoire of most, if not all insects, including those species living in normoxic environments [9]. A conserved common feature of the insect globins studied so far in more detail was their predominant expression in cells of the tracheal system and in the fat body $[9,14,18]$. Here we convincingly show that the two additional globin genes in the $D$. melanogaster genome, glob2 and glob3 [18], and also their single counterpart glob2/3 in D. virilis display an exceptional expression pattern, being almost exclusively transcribed in the adult male reproductive tract during several phases of spermatogenesis. Our qPCR and mRNA in situ hybridization results agree with a recent microarray study by Vibranovski et al. [28], which reported a malespecific expression of dmeglob2 and 3. In fact, that study suggested that dmeglob3 expression is stronger in post-meiotic compared to meiotic cell populations, whereas dmeglob2 exhibits a signal distribution characteristic for genes transcribed during meiosis.

Although globins are traditionally known as $\mathrm{O}_{2}$ supply proteins of the respiratory system, the expression of globins in the male reproductive system is not an entirely new fact. The vertebrate neuroglobin, for example, which is well recognized for its cell-protective role in nerve cells, is also expressed for yet unknown reasons in spermatogonia and primary spermatocytes of mouse testes [29]. Very recently, we reported the identification of a highly specialized chimeric protein of yet unknown function, in which a globin domain is fused to a protease-like domain [30]. This chimeric globin was named androglobin due to its predominant expression in testes tissue. While this new member of the globin family is evolutionary ancient and extremely conserved, being present in vertebrates, other chordates, lophotrochozoa, ecdysozoa, more basal animal clades and even choanoflagellates, the available genomes of Drosophila do not contain an androglobin ortholog [30]. It is therefore tempting to speculate that flies have evolved the glob2 and glob3 genes to compensate for a loss of their androglobin lineage. Since glob2 and glob3 are single-domain globins without a fused protease part, they would under this scenario have to interact via their $\mathrm{N}$ - and $\mathrm{C}$-terminal extensions [18] with other partner proteins to mimic the role of androglobin.

With the findings for neuroglobin, androglobin and Drosophila glob2 and glob3, the expression of globins in the male reproductive system and, more specifically, in the process of spermatogenesis appears to reflect an important aspect of globin function. Over the last decade, the traditional globin functions in $\mathrm{O}_{2}$ supply and storage $[31,32]$ have been complemented by other, equally important physiological roles, e.g. in the detoxification of harmful reactive oxygen species [ROS; [33-35]], in the scavenging and/or production of the bio-active gas nitric oxide [NO; $[36,37]]$, in redox-mediated cell signaling and apoptosis regulation [38] and in lipid metabolism $[39,40]$. Currently, we have only indirect data to distinguish between these possibilities for glob2 and glob3. However, we consider a conventional role of glob2 and glob3 in $\mathrm{O}_{2}$ supply in testes rather unlikely in light of the observed transcriptional down-regulation of both genes after experimental hypoxia, while traditional hemoglobins like those in the midge Chironomus or the crustacean Daphnia magna increase expression under hypoxic conditions $[8,41,42]$. Alternatively, Drosophila glob2 and glob3 may be instrumental in alleviating oxidative stress in the male reproductive tract, which is routinely exposed to ROS formed as by-products during oxidative metabolism. Spermatozoa are particularly sensitive to oxygen-induced damage mediated by lipid peroxidation $[43,44]$. This sensitivity is enforced by the shortage of antioxidants in the spermatozoa $[45,46]$. The importance of an effective antioxidants system for correct spermatogenesis in Drosophila has been shown e.g. in male flies with a null mutation in the gene for $\mathrm{Cu}-\mathrm{Zn}$ superoxide dismutase, which leads to a reduced fertility among other phenotypic abnormalities $[47,48]$. While exposure of flies to oxidative stress was accompanied by an upregulation of antioxidant genes [49], we could not detect such a response for dmeglob2 and 3 after experimental hyperoxia. Thus, currently no correlative evidence for an involvement of glob2 and glob3 in ROS detoxification exists. Modulation of the glob2 and 3 activity by transgenic overexpression or RNAi-mediated knockdown in vivo will be instrumental in testing these functional hypotheses. Unfortunately, biophysical data on the ligand binding characteristics of the two proteins are still lacking, because recombinant expression was not successful up to now due to the tendency of both $D$. melanogaster glob2 and glob3 to form stable precipitates. The primary sequences of both globins, however, harbour all key residues required for heme- and gas ligand-binding [18]. 
Molecular evolution of the Drosophila globin gene family: a complex scenario of neo- and subfunctionalization?

The highly specialized expression pattern of glob2 and glob3 has to be interpreted in the light of the evolutionary history of the Drosophila globin gene family. With the notable exception of the very large globin gene family of chironomids, which provides the midge larvae with huge amounts of extracellular globins for survival in hypoxic aquatic habitats, all other insect taxa studied so far on the genome sequence level have only one ( $B$. mori), two (A. mellifera, A. aegypti, A. gambiae) or three (Drosophila spec.) globin genes $[9,12]$. Molecular phylogenetic reconstructions based on globin amino acid sequence data have shown that Drosophila glob1 forms a clade with the globins of chironomids and the horse botfly G. intestinalis [18]. Glob1 is thus representative for a class of presumably $\mathrm{O}_{2}$-supplying globins from brachyceran and nematoceran dipterans. In contrast, glob2 and glob3 appeared at a basal position in the globin phylogenetic tree, with no clear affiliations to other insect globins [18]. Evolutionary rate calculations ([18]; this paper) show that glob2 and glob3 have evolved about threefold faster than glob1, so that the ancestral tree position might result from a long-branch attraction artefact. Instead, the absence of glob2-glob3 orthologs in other insect genome sequences (our unpublished observations) strongly suggests that this testes-specific globintype has evolved secondarily in the ancestor of the Drosophila genus. This derived evolutionary origin of glob2glob3 would be in agreement with the idea that they compensate for the taxon-specific loss of the testesexpressed androglobin gene lineage.

Gene synteny data enabled us to reconstruct the history of glob2 and 3 within the genus. We identified glob2 and glob3 orthologs in the nine species, which belong to the Sophophora subgenus, whereas the three species of the Drosophila subgenus harbour only a single cognate gene (glob2/3). Given the widely accepted phylogenetic relations among the twelve species [19], this distribution suggests the duplication of the glob2/3 gene in the stem lineage of the Sophophora subgenus and thus before the radiation of this clade at least 40 million years ago [20]. This 'glob2/3 duplication-in-Sophophora subgenus' scenario is more parsimonious than the alternative 'glob2 or 3 deletion-in-Drosophila subgenus' hypothesis, which implies the duplication of a testis-globin ancestor before the radiation of the Drosophila genus and the subsequent loss of one of the paralogs in the stem lineage of the Drosophila subgenus. Our tree reconstructions and hypothesis testing are fully in line with the more parsimonious interpretation of the glob2 and glob3 history in the sampled species. The finding of introns in all gene copies suggests that the duplication of the glob2/3 gene resulted from unequal crossing over, rather than retrotransposition. Probably, the duplication event in the Sophophora ancestor initially resulted in a head-to-tail orientation of glob2 and glob3 on a chromosome arm, which is equivalent to arm $3 \mathrm{R}$ in $D$. melanogaster (event A, Figure 1). This situation is today conserved in D. ananassae, D. pseudoobscura and $D$. persimilis. In $D$. willistoni the glob2 ortholog has most probably been transposed onto chromosomal arm 2R, equivalent to $2 \mathrm{~L}$ in $D$. melanogaster (event B, Figure 1). This corresponds to an increased activity of transposable elements in the D. willistoni [19]. Likewise, the separation of glob2 and glob3 by about $800 \mathrm{~kb}$ in the representatives of the $D$. melanogaster subgroup probably has been caused by a subsequent transposition of the glob2 paralog (event D, Figure 1).

The evolutionary fate of gene duplicates and the consequences of gene duplication for the evolution of novel adaptive traits are matters of intense research and debate [50]. Early studies [e.g. [51]] have contrasted the alternative models of neofunctionalization (i.e. one gene copy stays conserved, the other evolves a novel function) and subfunctionalization (i.e. both gene copies loose part of their functions and have to complement each other). However, ongoing research revealed that evolutionary scenarios of some if not most gene copies might be more complex than anticipated before [52]. Regarding the glob2 and glob3 gene pair as a possible model case for connecting molecular evolution to the phenotype, we applied codon-based maximum likelihood analyses of sequence evolution, calculating ratios of non-synonymous to synonymous nucleotide substitutions $\left(d_{\mathrm{N}} / d_{\mathrm{S}}\right)$, at the codon- and branch-site level to infer selective regimes acting on the globin gene duplicates. The 'glob2/3 duplication-in-Sophophora subgenus' hypothesis, supported by both, parsimony criteria and hypothesis testing, served as the basis for these analyses.

It is well-known that genes encoding proteins involved in sex and reproduction are often subjected to a wide array of selective forces like sexual conflict and male competition [53,54] and actually show signatures of positive selection and adaptive evolution [19,55-59]. Considering an exclusive expression of $D$. virilis and $D$. melanogaster glob2 and glob3 in the male genital tract, present evidence from codon-specific analyses for strong negative selection $\left(d_{\mathrm{N}} / d_{\mathrm{S}}=0.141\right)$ of Drosophila glob2/3, glob2, and glob3 is unexpected and points to high levels of functional constraint. Against this background, it appears remarkable that an estimated proportion of $42 \%$ of codon sites experienced strong positive selection $\left(d_{\mathrm{N}} /\right.$ $d_{\mathrm{S}}=5.642$ ) along the glob2 stem lineage (Figure 2). Taking additionally into account the likely absence of a positively selected extra site class along the glob3 stem lineage, present observations are not in line with the duplication > degeneration > complementation or 
subfunctionalization models [51,54-56], nor with the recently introduced model of subneofunctionalization [52]. These models consistently assume similar evolutionary fates of the two gene copies following gene duplication, either as a consequence of relaxed functional constraint [51] or by a succession of relaxed functional constraint and positive selection along both lineages [52]. Present observations rather remind of the classical concept of neofunctionalization sensu Ohta [57], assuming the conservation of one gene copy by negative selection, while the paralog evolves a new function under the influence of positive selection. Although the adaptive value of the positively selected amino acid exchanges along the glob2 stem lineage has still to be elucidated, results of the present sequence analyses suggest at least some functional differentiation of Sophophora glob2 compared to Sophophora glob3. On the other hand, the glob2 and glob3 genes in the Sophophora species appear to complement each other in terms of quantitative and regional RNA expression patterns, which is consistent with a subfunctionalization regime. A complex mixture of changing selective constraints over evolutionary time may therefore be a realistic alternative to simple models of gene duplicate evolution.

\section{Methods}

Sequence retrieval, gene phylogeny, molecular evolution and promoter prediction

Coding sequences of Drosophila glob2, glob3 and glob2/ 3 genes were extracted from Flybase http://flybase.org/. Genomic loci were identified by BLASTN search [60], using coding sequences as query. The dataset contained 33 glob1, glob2, glob3 and glob2/3 sequences of 9 representatives of the Sophophora subgenus and 3 representatives of the Drosophila subgenus plus glob1 of Gasterophilus intestinalis and hemoglobin CTTIII of Chironomus thummi thummi as outgroup representatives. Globin nucleotide sequences were aligned in the amino acid mode using the ClustalX algorithm implemented in BioEdit. For phylogenetic purposes, the highly divergent 5' and 3' ends of the proteins were trimmed, resulting in an alignment of $447 \mathrm{bp}$, comprising the globin domain.

We tested for potential gene conversion between paralogs at the nucleotide level using Geneconv version 1.81 $[61,62]$, which screens alignments for similar segments in the pairwise comparison suggestive of past gene conversion. To estimate the rates of amino acid sequence evolution of Drosophila glob1, glob2 and glob3, pairwise protein distances were calculated using the program MatGat http://bitincka.com/ledion/matgat/ and applying the PAM matrix. We followed the accepted divergence estimates of drosophilid taxa as proposed by Tamura et al. [20].
In order to infer the phylogenetic relations among glob1, glob2, glob3 and glob2/3 tree reconstructions were carried out at the nucleotide level using a maximum likelihood (ML) approach and Bayesian phylogenetic inference. To minimize the possible influence of saturation on tree reconstruction, only first and second codon positions were used. Applying the Akaike Information Criterion and assuming 4 rate categories, Treefinder [63] identified a special case of the GTR model, called J1[Optimum, Empirical]:G[Optimum]:4 in the Treefinder terminology, as the model of best fit. The J1 model assumes the same rates for i) TA and TG substitutions, and ii) CA and CG substitutions. Except for this constraint, base frequencies, substitution rates and gamma shape parameter were freely estimated from the data. Branch support was estimated by Local Rearrangements of tree support-Expected Likelihood Weights (LRELWs) [64] on the basis of 10.000 replicates. The Bayesian analysis was performed using MrBayes v3.1.2 $[65,66]$. As the J1 model cannot be specified in MrBayes, we assumed the original GTR model instead. We used 2 independent runs, each with four chains and 1.000.000 generations, discarding the first 200.000 generations as 'burnin'. Trees were edited using TreeView [67]. We further elucidated the phylogeny within a clade uniting all glob2, glob3 and glob2/3 sequences. Therefore, parametric bootstrap testing was performed specifying a topology reflecting the 'glob2/3 duplication-in-Sophophora subgenus' scenario as alternative hypothesis $\mathrm{H} 1$ and a topology reflecting the 'glob2 or 3 deletion-in-Drosophila subgenus' as null hypothesis ( $\mathrm{H0})$. At the ortholog level, both trees followed the generally accepted phylogeny within the Drosophila genus [19].

We employed the ML framework implemented in CODEML (PAML v4.4 package) [68] to test for signatures of positive selection using the ratio of non-synonymous to synonymous nucleotide substitution rates $(\omega=$ $\left.d_{\mathrm{N}} / d_{\mathrm{S}}\right)$ as a measure. In order to minimize the potential effect of saturation on results, analyses of sequence evolution were carried out based on a restricted dataset of 447 bp comprising Drosophila glob2/3, Sophophora glob2 and Sophophora glob3 sequences (altogether 21 sequences) and the intree shown in Figure 2. In a first approach, we tested for the presence of candidate codon sites of positive selection across the alignment. In detail, we compared the fit of models M7 (beta) and M8 (beta plus $\omega)$ per likelihood ratio test (LRTI). M7 and M8 describe the codon distribution in the $\omega$ interval $(0,1)$ as a beta function. However, while M7 confines $\omega$ to $(0$, 1), M8 allows for a positively selected extra site class. To avoid local optima, we ran M8 twice with different initial $\omega$ values $(0.6$ and 1.6). For LRTI, twice the log likelihood difference $(2 \Delta l)$ between the nested models was compared to critical values (cv) from a chi-square 
distribution with degrees of freedom (df) equal to the difference in the number of free parameters between the models, which is 4-2 $=2$.

We moreover tested for the presence of positively selected codon sites across user-defined "foreground" branches (branch-site LRTII). In detail, we compared the fit of two versions of modified branch-site model A [69]. Both model versions assume a negatively selected site class and a neutrally evolving site class across the entire phylogeny. Both versions further distinguish a third site class with $d_{\mathrm{N}} / d_{\mathrm{S}}<1$ or $d_{\mathrm{N}} / d_{\mathrm{S}}=1$ across the background. However, while $d_{\mathrm{N}} / d_{\mathrm{S}}$ of the third site class is fixed at 1 across the foreground in the null version of model A, the alternative version allows for a foreground $d_{\mathrm{N}} / d_{\mathrm{S}}>1$. We alternatively defined the Sophophora glob2 and the Sophophora glob3 stem lineages as foreground branches. For LRTII, $2 \Delta l$ between the two model versions was compared to $\mathrm{cv}$ from a 50:50 mixture of a point mass at zero and a chi-square distribution with $d f=1$, which are 2.71 and 3.84 at the $5 \%$ and $2.5 \%$ levels of significance. Strict Bonferroni adjustment was performed to adjust for twofold testing, thus lowering the $5 \%$ level of significance to $2.5 \%$.

The identification of possible binding-sites of RNA polymerase II was performed with the program Neural Network Promoter Prediction (NNPP) on the Berkeley Drosophila Genome Project homepage http://www.fruitfly.org/seq_tools/promoter.html. The NNPP program recognizes RNA polymerase II promoter via a TATA box and the Initiator transcription start site $[23,24]$. Additionally, we used the hidden Markov model-based program McPromoter http://tools.igsp.duke.edu/generegulation/McPromoter/[21,22] with the genomic sequences of D. melanogaster glob2, glob3, and D. simulans and D. sechellia glob3 as input.

\section{Fly stocks}

Hypoxia experiments were carried out using D. melanogaster wild type strain Oregon R. In situ hybridization and developmental stage-specific glob2, glob3 and glob2/ 3 mRNA expression analyses were carried out using $D$. melanogaster wild type strain Oregon $\mathrm{R}$ and $D$. virilis. Flies were kept on standard cornmeal agar at $25^{\circ} \mathrm{C}$.

\section{Hypoxia and hyperoxia experiments}

25 adult $D$. melanogaster were experimentally exposed to moderate hypoxia $\left(5 \% \mathrm{O}_{2}\right.$ for $\left.24 \mathrm{~h}\right)$, severe hypoxia $\left(1 \% \mathrm{O}_{2}\right.$ for $1 \mathrm{~h}, 3 \mathrm{~h}$ and $\left.4 \mathrm{~h}\right)$ and hyperoxia $\left(95 \% \mathrm{O}_{2}\right.$ for $12 \mathrm{~h}$ ), using a translucent PRO-OX chamber (BioSpherix Ltd., New York, USA). During hypoxia treatments, animals were monitored for vitality and behavioural reactions that are known being caused by the applied $\mathrm{O}_{2}$ concentrations (see e.g. [70]. Flies were kept at $25^{\circ} \mathrm{C}$ at the pre-adjusted $\mathrm{O}_{2}$ concentration that were obtained by mixing nitrogen or oxygen with ambient air. Technical nitrogen and oxygen were obtained from Westfalen AG (Münster, Germany). Gas concentrations were measured and kept constant by an oxygen sensor (E-702, BioSpherix, Ltd., New York, USA). After the desired time, animals were collected and immediately shock-frozen in liquid nitrogen. Samples were stored at $-80^{\circ} \mathrm{C}$ until use.

\section{RNA preparation and quantitative real-time reverse transcription-PCR (qPCR)}

Total RNA was isolated using the RNeasy Mini Kit (Qiagen, Hilden, Germany), quantified and checked on integrity. Reverse transcription was carried out with 1 $\mu \mathrm{g}$ total RNA employing the Superscript II RNase $\mathrm{H}^{-}$ reverse transcriptase (Invitrogen, Karlsruhe, Germany). qPCR experiments were carried out on the ABI Prism 7500 Sequence Detection System (Applied Biosystems, Darmstadt, Germany). For primer sequences, see Additional File 5. mRNA expression levels were calculated by the standard-curve approach, measuring the Ct-values. Factors of differential gene expression in developmental stages were calculated relative to the gene expression in adult males. D. melanogaster glob2, glob3 and lactate dehydrogenase $(L D H)$ expression data under different oxygen conditions were normalized relative to expression of the ribosomal protein gene $L 17 A$, which is not regulated by hypoxia according to microarray experiments (B. Adryan and R. Schuh, Göttingen, personal communication). Factors of differential gene regulation were calculated relative to the normoxic condition $(21 \%$ $\mathrm{O}_{2}$ ). Statistical evaluation was performed by calculating the mean values of the factors of regulation and their standard deviation. Two independent experiments (biological replicates) were performed for each condition, and each qPCR assay was run in duplicate. The significance of the data was assessed by a two-tailed Student's $t$-test employing the Microsoft Excel spreadsheet program.

\section{Preparation of genomic DNA and genomic PCR}

Genomic DNA was isolated from 20 L3 larvae according to the protocol by Huang et al. [71]. Genomic PCR was performed using Taq polymerase (Sigma-Aldrich Chemie $\mathrm{GmbH}$, Munich, Germany) according to the manufacturer's recommendations (for primer sequences, see Additional File 7).

\section{mRNA in situ hybridization}

mRNA probes for in situ hybridization experiments were prepared by in vitro transcription from the coding sequence of $D$. melanogaster glob2, glob3 and D. virilis glob2/3 using the digoxigenin RNA Labelling Kit (Roche Applied Science, Mannheim, Germany). The D. melanogaster $\beta$-tubulin $85 \mathrm{D}$ was used as a positive control for 
testes-specific mRNA expression [72]. It revealed the expected staining of cells in meiosis II (not shown). In situ hybridization to whole testes was carried out according to the protocol from Tautz and Pfeifle [73] with the following changes: testes were dissected in testes buffer [74-76], fixed with $4 \%$ formaldehyde/PBS for $30 \mathrm{~min}$ and washed briefly in PBS $+0.1 \%(\mathrm{v} / \mathrm{v})$ Tween-20. Tissues were pre-incubated with hybridization solution $(50 \%[\mathrm{v} / \mathrm{v}] 10 \mathrm{x}$ Diethylpyrocarbonate-SSC, $50 \%[\mathrm{v} / \mathrm{v}]$ formamide, $0.1 \%[\mathrm{v} / \mathrm{v}]$ Tween-20) containing $0.1 \mathrm{mg} / \mathrm{ml}$ salmon sperm carrier DNA at $55^{\circ} \mathrm{C}$. Hybridization was carried out at $55^{\circ} \mathrm{C}$ overnight in hybridization solution containing $0.1 \mathrm{mg} / \mathrm{ml}$ salmon sperm DNA and $0.5 \mu \mathrm{g} / \mathrm{mL}$ antisense probe. Washing was performed in PBS $+0.1 \%(\mathrm{v} / \mathrm{v})$ Tween -20 at $65^{\circ} \mathrm{C}$. Detection and staining were carried out using an anti-DIG alkaline phosphatase-conjugated antibody and NBT/BCIP solution (Roche Applied Science, Mannheim, Germany) according to the manufacturer's recommendations.

\section{Additional material}

\section{Additional file 1: Designations of Drosophila globin genes.}

Additional file 2: Phylogenetic relationship of Drosophila globins

Phylogenetic reconstruction of Drosophila glob1, glob2, glob3 and glob2/3 including G. intestinalis glob1 (ginglob1) and C. thummi thummi Hblll (cttHbllI) at the nucleotide level (using only first and second codon positions). (A) by applying a Maximum likelihood approach implemented in Treefinder and (B) a Bayesian analysis using MrBayes.

Additional file 3: Absolute mRNA quantitation of dmeglob2, dmeglob3 and dviglob2/3. Glob2, glob3 and glob2/3 absolute mRNA copy number in male adult flies of $D$. melanogaster and $D$. virilis, measured with qPCR. Dmeglob2 (144784 copies) and dmeglob3 (150026 copies) copy number summed up is equal to dviglob2/3 copy number (311673 copies).

Additional file 4: Glob3 gene region with Jockey transposon. Schematic diagram of the genomic region of $D$. melanogaster glob3 including the transposable element inserted downstream of the glob3 gene in comparison to the corresponding genomic region of $D$. sechellia glob3. In D. melanogaster, the insertion and the putative duplicated sequences are indicated. In $D$. sechellia, the predicted promoter sequence spanning the transposon insertion sequence in $D$. melanogaster are highlighted. Exons and $5^{\prime} U T R$ and distances between $3^{\prime}$ end of transposable element and Exon1 in both D. melanogaster and D. sechellia are plotted.

Additional file 5: Negative control for mRNA in situ hybridization. As negative control for in situ hybridization, a sense mRNA probe of dmeglob2 was applied at otherwise identical hybridization conditions.

Additional file 6: Regulation of $L D H$ mRNA in D. melanogaster male adults after hypoxic stress. mRNA levels (bars) are shown relative to the gene expression at normoxia (21\%). (A) $\mathrm{LDH}$ expression after $1 \% \mathrm{O}_{2}$ for $1 \mathrm{~h}, 3 \mathrm{~h}$ and $4 \mathrm{~h}$. After $1 \mathrm{~h}$ of hypoxia, no alteration in $L D H$ expression could be detected. $3 \mathrm{~h}$ of hypoxia caused the LDH mRNA levels to increase about 2.2 fold and $4 \mathrm{~h}$ of hypoxia to about 3.4 fold compared to the normoxic control (B) $L D H$ expression after $6 \% \mathrm{O}_{2}$ for $24 \mathrm{~h}$. After applying long-term moderate hypoxia with $6 \% \mathrm{O}_{2}$ for $24 \mathrm{~h}$, an increase in $L D H$ mRNA expression to about 1.5 fold could be detected $\left({ }^{*} \mathrm{p}<0.05\right.$; $\left.{ }^{* *} p<0.01\right)$.

Additional file 7: Oligonucleotides and PCR conditions

\section{Acknowledgements}

TH and TB gratefully acknowledge funding by the Deutsche

Forschungsgemeinschaft (DFG Ha2103/5 and Bu956/6). TH received additional funding by the University of Mainz Computational Sciences Center (SRFN). The authors would like to thank two anonymous reviewers for their very helpful remarks.

\section{Author details}

Institute of Molecular Genetics, University of Mainz, 55099 Mainz, Germany. ${ }^{2}$ Center for Systems Biology, University of Freiburg, 79104 Freiburg, Germany. ${ }^{3}$ Institute of Anthropology, University of Mainz, 55099 Mainz, Germany. ${ }^{4}$ Biocenter Grindel and Zoological Museum, University of Hamburg, 20146 Hamburg, Germany.

\section{Authors' contributions}

EG participated in the design of the study, carried out QPCR and mRNA in situ hybridization experiments, extracted the glob2, glob3 and glob2/3 genes in the available sequenced Drosophila genomes and analyzed their genomic organization, calculated the evolutionary rates, performed the promoter search, participated in the sequence alignments, phylogenetic reconstructions and in the analyses regarding molecular evolution and drafted the manuscript. HH participated in the sequence alignments, phylogenetic reconstructions, in the analyses regarding molecular evolution and helped to draft the manuscript. SZ participated in GPCR and mRNA in situ hybridization experiments and helped to draft the manuscript. TB helped to draft the manuscript. TH conceived the study, participated in its design and coordination, and helped to draft the manuscript. All authors read and approved the final manuscript.

Received: 9 October 2011 Accepted: 19 March 2012 Published: 19 March 2012

\section{References}

1. Brusca RC, Brusca GJ: Invertebrates Sunderland, MA: Sinauer; 1990.

2. Willmer P, Stone G, Johnston IA: Physiology of Animals Oxford UK: Blackwell Press; 2000.

3. Vinogradov SN, Hoogewijs D, Bailly X, Arredondo-Peter R, Guertin M, Gough J, Dewilde S, Moens L, Vanfleteren JR: Three globin lineages belonging to two structural classes in genomes from the three kingdoms of life. Proc Natl Acad Sci USA 2005, 102:11385-11389.

4. Keilin D, Wang YL: Haemoglobin of Gastrophilus larvae. Purification and properties. Biochem J 1946, 40:855-866.

5. Dewilde S, Blaxter M, Van Hauwaert ML, Van Houte K, Pesce A, Griffon N, Kiger L, Marden MC, Vermeire S, Vanfleteren J, et al: Structural, functional, and genetic characterization of Gastrophilus hemoglobin. J Biol Chem 1998, 273:32467-32474

6. Matthews PG, Seymour RS: Diving insects boost their buoyancy bubbles. Nature 2006, 441:171

7. Ewer RF: On the function of hemoglobin in Chironomus. Journal of Experimental Biology 1942, 18:197-205.

8. Osmulski PA, Leyko W: Structure, function and physiological role of Chironomus hemoglobin. Comparative Biochemistry and Physiology 1985, 85B:701-722.

9. Burmester T, Hankeln T: The respiratory proteins of insects. J Insect Physiol 2007, 53:285-294.

10. Hankeln T, Klawitter S, Kramer M, Burmester T: Molecular characterization of hemoglobin from the honeybee Apis mellifera. J Insect Physiol 2006, 52:701-710.

11. Burmester $\mathrm{T}$, Klawitter $\mathrm{S}$, Hankeln $\mathrm{T}$ : Characterization of two globin genes from the malaria mosquito Anopheles gambiae: divergent origin of nematoceran haemoglobins. Insect Mol Biol 2007, 16:133-142.

12. Kawaoka S, Katsuma S, Meng Y, Hayashi N, Mita K, Shimada T: Identification and characterization of globin genes from two lepidopteran insects, Bombyx mori and Samia cynthi ricini. Gene 2009, 431:33-38.

13. Burmester T, Hankeln T: A globin gene of Drosophila melanogaster. Mol Biol Evol 1999, 16:1809-1811.

14. Hankeln T, Jaenicke V, Kiger L, Dewilde S, Ungerechts G, Schmidt M, Urban J, Marden MC, Moens L, Burmester T: Characterization of Drosophila hemoglobin. Evidence for hemoglobin-mediated respiration in insects. J Biol Chem 2002, 277:29012-29017. 
15. de Sanctis D, Dewilde S, Vonrhein C, Pesce A, Moens L, Ascenzi P, Hankeln T, Burmester T, Ponassi M, Nardini M, Bolognesi M: Bishistidyl heme hexacoordination, a key structural property in Drosophila melanogaster hemoglobin. J Biol Chem 2005, 280:27222-27229.

16. Gorr TA, Tomita T, Wappner P, Bunn HF: Regulation of Drosophila hypoxiainducible factor (HIF) activity in SL2 cells: identification of a hypoxia-induced variant isoform of the HIFalpha homolog gene similar. J Biol Chem 2004, 279:36048-36058.

17. Gleixner E, Abriss D, Adryan B, Kraemer M, Gerlach F, Schuh R, Burmester T, Hankeln T: Oxygen-induced changes in hemoglobin expression in Drosophila. Febs J 2008, 275:5108-5116.

18. Burmester T, Storf J, Hasenjager A, Klawitter S, Hankeln T: The hemoglobin genes of Drosophila. Febs J 2006, 273:468-480

19. Clark AG, Eisen MB, Smith DR, Bergman CM, Oliver B, Markow TA, Kaufman TC, Kellis M, Gelbart W, lyer VN, et al: Evolution of genes and genomes on the Drosophila phylogeny. Nature 2007, 450:203-218.

20. Tamura K, Subramanian S, Kumar S: Temporal patterns of fruit fly (Drosophila) evolution revealed by mutation clocks. Mol Biol Evol 2004, 21:36-44.

21. Ohler U: Identification of core promoter modules in Drosophila and their application in accurate transcription start site prediction. Nucleic Acids Res 2006, 34:5943-5950.

22. Ohler U, Liao GC, Niemann H, Rubin GM: Computational analysis of core promoters in the Drosophila genome. Genome Biol 2002, 3, RESEARCH0087.

23. Reese MG: Application of a time-delay neural network to promoter annotation in the Drosophila melanogaster genome. Comput Chem 2001, 26:51-56.

24. Reese MG, Eeckman FH, Kulp D, Haussler D: Improved splice site detection in Genie. J Comput Biol 1997, 4:311-323.

25. Douglas RM, Farahani R, Morcillo P, Kanaan A, Xu T, Haddad GG: Hypoxia induces major effects on cell cycle kinetics and protein expression in Drosophila melanogaster embryos. Am J Physiol Regul Integr Comp Physiol 2005, 288:R511-521.

26. Camenisch G, Stroka DM, Gassmann M, Wenger RH: Attenuation of HIF-1 DNA-binding activity limits hypoxia-inducible endothelin-1 expression. Pflugers Arch 2001, 443:240-249.

27. Loots GG, Ovcharenko I, Pachter L, Dubchak I, Rubin EM: rVista for comparative sequence-based discovery of functional transcription factor binding sites. Genome Res 2002, 12:832-839.

28. Vibranovski MD, Lopes HF, Karr TL, Long M: Stage-specific expression profiling of Drosophila spermatogenesis suggests that meiotic sex chromosome inactivation drives genomic relocation of testis-expressed genes. PLoS Genet 2009, 5:e1000731.

29. Reuss S, Saaler-Reinhardt S, Weich B, Wystub S, Reuss MH, Burmester T, Hankeln T: Expression analysis of neuroglobin mRNA in rodent tissues. Neuroscience 2002, 115:645-656.

30. Hoogewijs D, Ebner B, Germani F, Hoffmann FG, Fabrizius A, Moens L, Burmester T, Dewilde S, Storz JF, Vinogradov SN, Hankeln T: Androglobin, a new metazoan family of chimeric globins with a $\mathrm{N}$-terminal calpain-like protease domain, preferentially expressed in vertebrate testes. Mol Biol Evol 2011

31. Dickerson RE, Geis I: Hemoglobin: structure, function, evolution, and pathology. Benjamin/Cummings Publ Co, Menlo Park, Calif 1983.

32. Wittenberg JB: Myoglobin-facilitated oxygen diffusion: role of myoglobin in oxygen entry into muscle. Physiol Rev 1970, 50:559-636.

33. Merx MW, Flogel U, Stumpe T, Godecke A, Decking UK, Schrader J: Myoglobin facilitates oxygen diffusion. FASEB J 2001, 15:1077-1079.

34. Flogel U, Godecke A, Klotz LO, Schrader J: Role of myoglobin in the antioxidant defense of the heart. FASEB J 2004, 18:1156-1158.

35. Weber RE, Fago A: Functional adaptation and its molecular basis in vertebrate hemoglobins, neuroglobins and cytoglobins. Respir Physiol Neurobiol 2004, 144:141-159.

36. Flogel U, Merx MW, Godecke A, Decking UK, Schrader J: Myoglobin: A scavenger of bioactive NO. Proc Natl Acad Sci USA 2001, 98:735-740.

37. Gladwin MT, Crawford JH, Patel RP: The biochemistry of nitric oxide, nitrite, and hemoglobin: role in blood flow regulation. Free Radic Biol Med 2004, 36:707-717

38. Fago A, Mathews AJ, Moens $L$, Dewilde S, Brittain T: The reaction of neuroglobin with potential redox protein partners cytochrome b5 and cytochrome c. FEBS Lett 2006, 580:4884-4888.
39. Sriram $R$, Kreutzer $U$, Shih $L$, Jue $T$ : Interaction of fatty acid with myoglobin. FEBS Lett 2008, 582:3643-3649.

40. Reeder BJ, Svistunenko DA, Wilson MT: Lipid binding to cytoglobin leads to a change in haem co-ordination: a role for cytoglobin in lipid signalling of oxidative stress. Biochem J 2011, 434:483-492.

41. Zeis B, Becher B, Goldmann T, Clark R, Vollmer E, Bolke B, Bredebusch I, Lamkemeyer T, Pinkhaus O, Pirow R, Paul RJ: Differential haemoglobin gene expression in the crustacean Daphnia magna exposed to different oxygen partial pressures. Biol Chem 2003, 384:1133-1145.

42. Gorr TA, Cahn JD, Yamagata H, Bunn HF: Hypoxia-induced synthesis of hemoglobin in the crustacean Daphnia magna is hypoxia-inducible factor-dependent. J Biol Chem 2004, 279:36038-36047.

43. Jones R, Mann T, Sherins R: Peroxidative breakdown of phospholipids in human spermatozoa, spermicidal properties of fatty acid peroxides, and protective action of seminal plasma. Fertil Steril 1979, 31:531-537.

44. Sanocka D, Kurpisz M: Reactive oxygen species and sperm cells. Reprod Biol Endocrinol 2004, 2:12.

45. Aitken RJ, Baker MA: Oxidative stress and male reproductive biology. Reprod Fertil Dev 2004, 16:581-588.

46. Aitken RJ, Buckingham DW, Carreras A, Irvine DS: Superoxide dismutase in human sperm suspensions: relationship with cellular composition, oxidative stress, and sperm function. Free Radic Biol Med 1996, 21:495-504.

47. Phillips JP, Campbell SD, Michaud D, Charbonneau M, Hilliker AJ: Null mutation of copper/zinc superoxide dismutase in Drosophila confers hypersensitivity to paraquat and reduced longevity. Proc Natl Acad Sci USA 1989, 86:2761-2765.

48. Parkes TL, Kirby K, Phillips JP, Hilliker AJ: Transgenic analysis of the CSODnull phenotypic syndrome in Drosophila. Genome 1998, 41:642-651.

49. Landis GN, Abdueva D, Skvortsov D, Yang J, Rabin BE, Carrick J, Tavare S, Tower J: Similar gene expression patterns characterize aging and oxidative stress in Drosophila melanogaster. Proc Natl Acad Sci USA 2004 101:7663-7668.

50. Lynch M, Conery JS: The evolutionary fate and consequences of duplicate genes. Science 2000, 290:1151-1155.

51. Force A, Lynch M, Pickett FB, Amores A, Yan YL, Postlethwait J: Preservation of duplicate genes by complementary, degenerative mutations. Genetics 1999, 151:1531-1545.

52. He X, Zhang J: Rapid subfunctionalization accompanied by prolonged and substantial neofunctionalization in duplicate gene evolution. Genetics 2005, 169:1157-1164

53. Sella G, Petrov DA, Przeworski M, Andolfatto P: Pervasive natural selection in the Drosophila genome? PLOS Genet 2009, 5:e1000495.

54. Hughes AL: The evolution of functionally novel proteins after gene duplication. Proc Biol Sci 1994, 256:119-124.

55. Jensen RA: Enzyme recruitment in evolution of new function. Annu Rev Microbiol 1976, 30:409-425.

56. Orgel LE: Gene-duplication and the origin of proteins with novel functions. J Theor Biol 1977, 67:773.

57. Ohta T: Evolution by gene duplication and compensatory advantageous mutations. Genetics 1988, 120:841-847.

58. Herlyn $\mathrm{H}$, Zischler $\mathrm{H}$ : The molecular evolution of sperm zonadhesin. Int $J$ Dev Biol 2008, 52:781-790.

59. Nadeau NJ, Burke T, Mundy NI: Evolution of an avian pigmentation gene correlates with a measure of sexual selection. Proc Biol Sci 2007, 274:1807-1813

60. Altschul SF, Gish W, Miller W, Myers EW, Lipman DJ: Basic local alignment search tool. J Mol Biol 1990, 215:403-410.

61. Sawyer S: Statistical tests for detecting gene conversion. Mol Biol Evol 1989, 6:526-538.

62. Sawyer SA: GENECONV: A computer package for the statistical detection of gene conversion. Distributed by the author, Department of Mathematics, Washington University St Louis 1999 [http://math.wustl.edu/ sawyer].

63. Jobb G, von Haeseler A, Strimmer K: TREEFINDER: a powerful graphical analysis environment for molecular phylogenetics. BMC Evol Biol 2004, $4: 18$.

64. Strimmer K, Rambaut A: Inferring confidence sets of possibly misspecified gene trees. Proc Biol Sci 2002, 269:137-142.

65. Ronquist F, Huelsenbeck JP: MrBayes 3: Bayesian phylogenetic inference under mixed models. Bioinformatics 2003, 19:1572-1574. 
66. Huelsenbeck JP, Ronquist F, Nielsen R, Bollback JP: Bayesian inference of phylogeny and its impact on evolutionary biology. Science 2001, 294:2310-2314.

67. Page RD: Tree View: an application to display phylogenetic trees on personal computers. Comput Appl Biosci 1996, 12:357-358.

68. Yang Z: PAML 4: phylogenetic analysis by maximum likelihood. Mol Biol Evol 2007, 24:1586-1591.

69. Zhang J, Nielsen R, Yang Z: Evaluation of an improved branch-site likelihood method for detecting positive selection of the molecular level. Mol Biol Evol 2005, 22:2472-2479.

70. Wingrove JA, O'Farrell PH: Nitric oxide contributes to behavioral, cellular, and developmental responses to low oxygen in Drosophila. Cell 1999, 98:105-114.

71. Huang $A M$, Rehm EJ, Rubin GM: Quick preparation of genomic DNA from Drosophila. Cold Spring Harb Protoc 2009, 2009, pdb prot5198.

72. Bialojan S, Falkenburg D, Renkawitz-Pohl R: Characterization and developmental expression of beta tubulin genes in Drosophila melanogaster. Embo J 1984, 3:2543-2548.

73. Tautz D, Pfeifle C: A non-radioactive in situ hybridization method for the localization of specific RNAs in Drosophila embryos reveals translational control of the segmentation gene hunchback. Chromosoma 1989, 98:81-85.

74. Hennig W: Investigations of the structure and function of lampbrushYchromosome in spermatogenesis in Drosophila. Chromosoma 1967, 22:294-357.

75. Cenci G, Bonaccorsi S, Pisano C, Verni F, Gatti M: Chromatin and microtubule organization during premeiotic, meiotic and early postmeiotic stages of Drosophila melanogaster spermatogenesis. J Cell Sci 1994, 107(Pt 12):3521-3534.

76. Pisano C, Bonaccorsi S, Gatti M: The kl-3 loop of the $\mathrm{Y}$ chromosome of Drosophila melanogaster binds a tektin-like protein. Genetics 1993, 133:569-579.

doi:10.1186/1471-2148-12-34

Cite this article as: Gleixner et al:: Testes-specific hemoglobins in

Drosophila evolved by a combination of sub- and neofunctionalization after gene duplication. BMC Evolutionary Biology 2012 12:34.

\section{Submit your next manuscript to BioMed Central} and take full advantage of:

- Convenient online submission

- Thorough peer review

- No space constraints or color figure charges

- Immediate publication on acceptance

- Inclusion in PubMed, CAS, Scopus and Google Scholar

- Research which is freely available for redistribution

Submit your manuscript at www.biomedcentral.com/submit
Biomed Central 